\title{
Performance Evaluation of a Hybrid Photovoltaic/Thermoelectric Generator System under Non-Tracking and Tracking Condition
}

\author{
Sridhar Sripadmanabhan Indira ${ }^{1}$, Chockalingam Aravind Vaithilingam ${ }^{1 *}$, and Ramsundar \\ Sivasubramanian ${ }^{1}$ \\ ${ }^{1}$ Taylor's University, 1 Jalan Taylor's, 47500, Subang Jaya, Selangor, Malaysia
}

\begin{abstract}
In this study, the performance of the thermoelectric generator (TEG) modules in converting the excess photovoltaic (PV) temperature to electricity in a hybrid photovoltaic/thermoelectric (PV/TEG) generator system is investigated. A one-dimensional analytical heat transfer model for the hybrid PV/TEG with pin-fin based heat sink is developed in Engineering Equation Solver (EES) and simulated under tracking and nontracking conditions. The solar irradiance collected by the PV/TEG system under tracking and non-tracking conditions was calculated using the Solar Emulator tool via TracePro software. The effect of varying solar radiation and a varying number of TEGs on PV temperature, PV output, and TEG output is evaluated using solar radiation on March 15, 2020, in the Jalan Taylor's, Malaysia $\left(3.0626^{\circ} \mathrm{N}, 101.6168^{\circ} \mathrm{E}\right)$. Finally, the optimum number of TEG modules required for maximum TEG power output in the hybrid $\mathrm{PV} / \mathrm{TEG}$ system under tracking and non-tracking conditions is investigated and discussed. The maximum net $P_{T E G}$ is obtained for 336,339 , and 341 TEGs under no-tracking, single-axis, and dual-axis tracking conditions, respectively.
\end{abstract}

\section{Introduction}

Photovoltaic (PV) devices can directly convert solar energy into electricity. The main drawback of such devices is that a large portion of solar energy is absorbed as heat and affects the efficiency of the PV. Several active, passive cooling methods combined photovoltaic and thermal systems have been proposed and tested in past decades to improve efficiency. A straightforward way to use the waste heat from PV modules without mechanical parts is by using thermoelectric generators (TEGs). TEGs are solid-state devices that utilise the Seebeck effect to convert thermal energy (temperature gradient) into electricity [1], [2]. Several such hybrid systems have been reviewed recently [3-8]. Using thermoelectric generators to improve the efficiency of concentrated PV was first proposed by Xi and Swanson in 1979. Many hybrid PV/TEG systems have been studied recently [9-12].

\footnotetext{
${ }^{*}$ Corresponding author: aravindev@,ieee.org
} 
Bjork and Nielsen [13] found that the performance degradation of PV due to temperature is higher than the power produced by the TEG due to the low efficiency of TEG. Urbiola et al. [14] investigated TEG's performance in a solar hybrid system under concentration and non-concentration conditions. The results proved that TEG's inclusion in a hybrid concentrated PV system operating at higher temperature enhances the thermal stability of the system's electrical efficiency. Najafi et al. [15] modelled a combined PV/TEG system and calculated the optimum number of TEGs required for the system's maximum overall output power. Babu and Ponnambalam [9], in their study, theoretically evaluated the PV/TEG performance using commercial PV and TEG. The proposed configuration generates 5\% additional energy with an overall efficiency improvement of 6 $\%$ under standard test conditions. Li et al. [16] did an energy and exergy analysis of a PV/TEG hybrid system to evaluate its performance. The results showed that a high concentration ratio and suitable PV cell could improve the PV/TEG system's output efficiency. Marandi et al. [17] fabricated a solar cavity with hybrid PV/TEG modules and investigated its performance experimentally. The current and voltage of the cavity based PV/TEG system were three times greater than the flat PV/TEG system. Kutt et al. [18] optimised the number of TEG in a parabolic based concentrated solar TEG considering the electric power productivity for a full year.

The novelty of the present work is that a hybrid PV/TEG's performance and the optimum number of TEGs required for maximum output is evaluated for three operating conditions: without tracking, single-axis tracking, and dual-axis tracking. The PV/TEG yearly electric power generation is evaluated considering the annual solar radiation. The mathematical models of the hybrid PV and TEG system are used to optimise the number of TEGs integrated with the PV module based on TEG's output performance. The onedimensional energy transfer equations of the hybrid system are solved analytically in the EES environment.

\section{The Hybrid System Under Study}

The schematic of the hybrid PV/TEG system under study is shown in Fig. 1. The hybrid PV/TEG system consists of a PV layer integrated with TEGs on its rear side, and a pin-fin type heat sink is attached at the cold junction of TEG, which helps in cooling through natural convection. We consider a commercially available polycrystalline PV module (Kyocera KC 200GT) and bismuth telluride based TEG. The specifications and properties of the considered PV module, TEG module, and heat sink are presented in Table 1, Table 2 and Table 3, respectively. The solar radiation data for Jalan Taylor's geographical location, Malaysia $\left(3.0626^{\circ} \mathrm{N}, 101.6168^{\circ} \mathrm{E}\right)$, is deemed. The solar emulator tool in the TracePro software is used to obtain the hybrid system's annual solar radiation under no tracking, single-axis, and dual-axis tracking conditions.

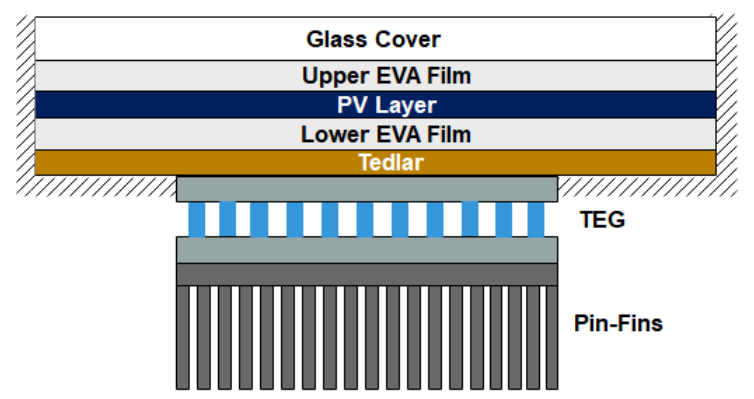

Fig. 1. The schematic of the hybrid PV/TEG system with pin-fin based heat sink understudy 
Table 1. The properties of PV used in the simulation

\begin{tabular}{|l|c|c|}
\hline \multicolumn{1}{|c|}{ Parameter } & Sign & Value \\
\hline Transmissivity of glass & $\tau_{g}$ & 0.95 \\
\hline Emissivity of glass & $\varepsilon_{g}$ & 0.85 \\
\hline Absorptivity of PV & $\alpha_{P V}$ & 0.9 \\
\hline Packaging factor & $\beta_{P V}$ & 0.93 \\
\hline Absorptivity of tedlar & $\alpha_{T}$ & 0.5 \\
\hline Thickness of glass & $L_{g}$ & $0.003 \mathrm{~m}$ \\
\hline Thickness of PV & $L_{P V}$ & $0.0002 \mathrm{~m}$ \\
\hline Thickness of EVA & $L_{E V A}$ & $500 \times 10^{-6} \mathrm{~m}$ \\
\hline Thickness of tedlar & $L_{\text {tedlar }}$ & $0.0003 \mathrm{~m}$ \\
\hline Thermal conductivity of glass & $K_{g}$ & $1.8 \mathrm{~W} / \mathrm{m} . \mathrm{K}$ \\
\hline Thermal conductivity of PV & $K_{P V}$ & $148 \mathrm{~W} / \mathrm{m} . \mathrm{K}$ \\
\hline Thermal conductivity of EVA & $K_{E V A}$ & $0.35 \mathrm{~W} / \mathrm{m} . \mathrm{K}$ \\
\hline Thermal conductivity of tedlar & $K_{\text {tedlar }}$ & $0.2 \mathrm{~W} / \mathrm{m} . \mathrm{K}$ \\
\hline Area of PV & $A_{P V}$ & $1.41075 \mathrm{~m}{ }^{2}$ \\
\hline Number of PV cells & - & $54(156 \mathrm{~mm} \mathrm{x} \mathrm{156mm)}$ \\
\hline PV efficiency under STC & $\eta_{\text {ref }}$ & 0.16 \\
\hline PV temperature coefficient & $\gamma$ & $0.004678 \mathrm{~K}-1$ \\
\hline Wind speed & $V$ & $1.8 \mathrm{~m} / \mathrm{s}$ \\
\hline
\end{tabular}

Table 2. Properties of the TEG used in the simulation

\begin{tabular}{|l|l|c|}
\hline \multicolumn{1}{|c|}{ Parameter } & \multicolumn{1}{|c|}{ Sign } & Value \\
\hline TEG area & $A_{\text {TEG }}$ & $30 \mathrm{~mm} \times 30 \mathrm{~mm}$ \\
\hline Thickness of ceramic & $L_{\text {ceramic }}$ & $0.0008 \mathrm{~m}$ \\
\hline Thermal conductivity of ceramic & $K_{\text {ceramic }}$ & $35 \mathrm{~W} / \mathrm{m} . \mathrm{K}$ \\
\hline Thickness of p and n leg & $L_{p} / L_{n}$ & $0.0016 \mathrm{~m}$ \\
\hline Thermal conductivity of p and n leg & $K_{p} / K_{n}$ & $1.82 \mathrm{~W} / \mathrm{m} . \mathrm{K}$ \\
\hline Seebeck coefficient of p and n leg & $\alpha_{p} / \alpha_{n}$ & $1.83 \times 10^{-4} \mathrm{~V} / \mathrm{K}$ \\
\hline Electrical resistivity of p and n leg & $\rho_{p} / \rho_{n}$ & $7.23 \times 10^{-6} \Omega . \mathrm{m}$ \\
\hline Number of pairs of p-n legs & $N_{p n}$ & 127 \\
\hline Sectional area of p-n legs & $A_{p} / A_{n}$ & $1 \mathrm{~mm}^{2}$ \\
\hline
\end{tabular}


Table 3. Properties of the Heat Sink used in the simulation

\begin{tabular}{|l|c|c|}
\hline \multicolumn{1}{|c|}{ Parameter } & Sign & Value \\
\hline Area of the fin base & $A_{b}$ & $30 \mathrm{~mm} \times 30 \mathrm{~mm}$ \\
\hline thermal conductivity of fin vase & $K_{b}$ & $200 \mathrm{~W} / \mathrm{m} . \mathrm{K}$ \\
\hline Thickness of fin base & $L_{b}$ & $0.003 \mathrm{~m}$ \\
\hline Number of Fins & $n_{\text {fin }}$ & 100 \\
\hline Diameter of fin & $D_{\text {fin }}$ & $0.0015 \mathrm{~m}$ \\
\hline Height of fin & $H_{\text {fin }}$ & $0.015 \mathrm{~m}$ \\
\hline thermal conductivity of fin & $K_{\text {fin }}$ & $200 \mathrm{~W} / \mathrm{m} . \mathrm{K}$ \\
\hline
\end{tabular}

\section{TracePro Simulation}

The photovoltaic panel is designed in Solidworks and then imported to TracePro for raytracing simulation. The top surface of the PV is defined as a perfect absorber $(100 \%$ absorptivity). Fig. 2 shows the sun source and the PV receiver modelled in the TracePro for ray-tracing. The sun's trajectory is set in the solar emulator tool using the location's geographical latitude and longitudinal values. Solar constant value is set as $1366 \mathrm{~W} / \mathrm{m}^{2}$, and Igawa all-sky model is considered with sky condition set as clear sky. Fig. 3 shows a screenshot of the graphical user interface of the Solar Emulator tool in TracePro. Around 2 million rays were used for ray simulation to obtain the average solar radiation received on the PV panel. Since only the average solar radiation is considered for the present study, the ray sensitivity test is not required in TracePro.

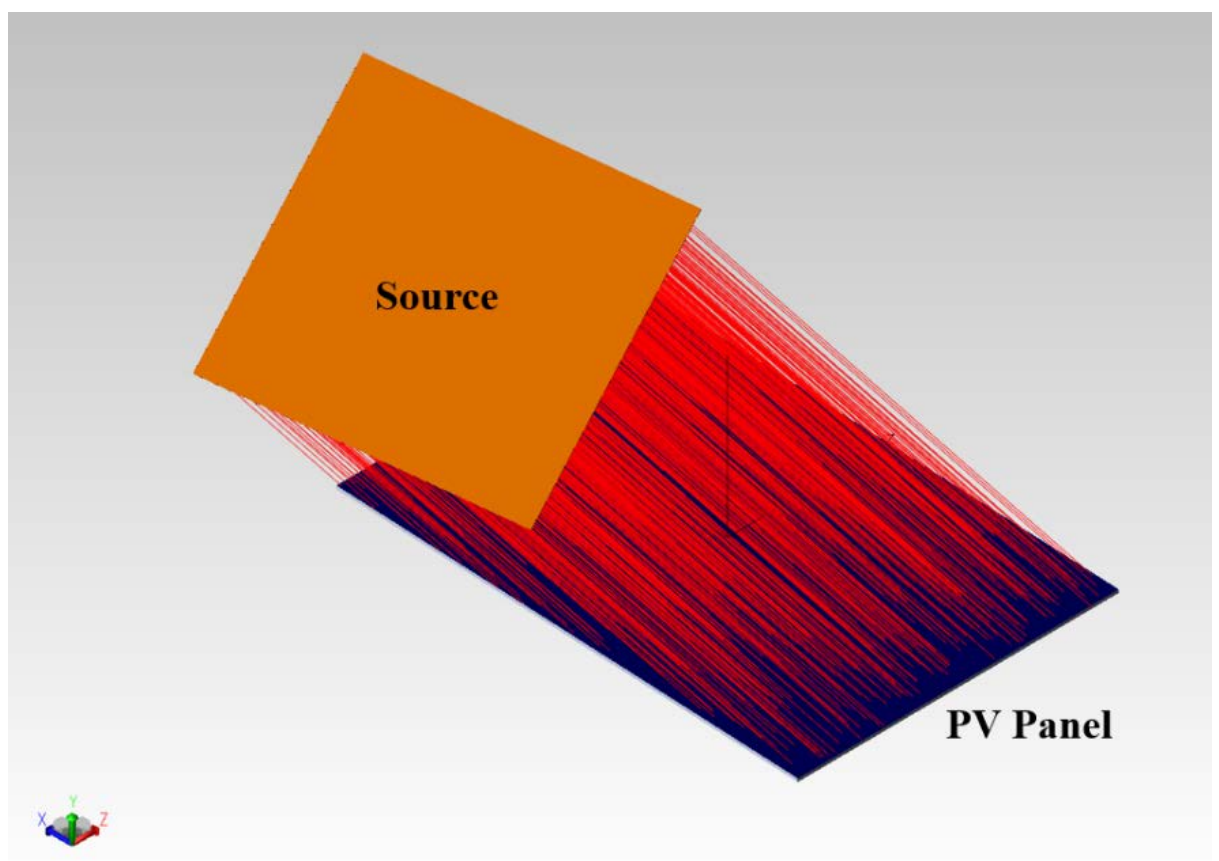

Fig. 2. Ray-tracing simulation of the PV panel in TracePro 


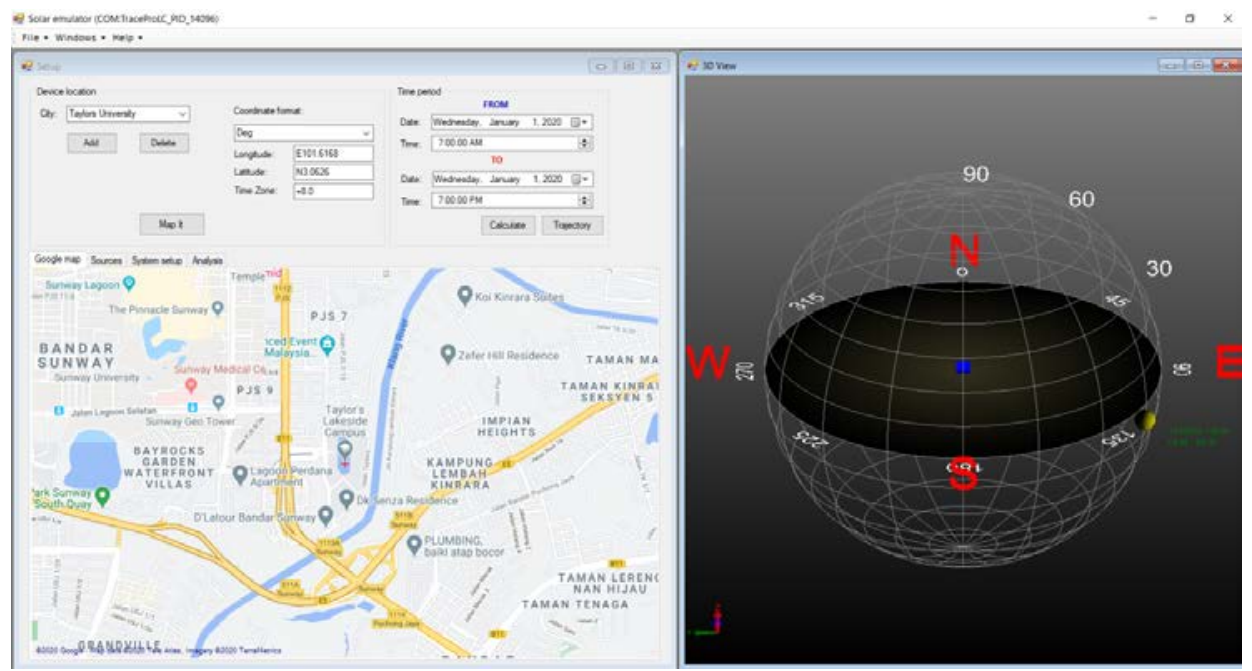

Fig. 3. The graphical user interface of the Solar Emulator tool in TracePro showing the location setup and 3D view of the sun's trajectory

\section{Mathematical Modelling}

The energy transfer network of the PV/TEG system under study based on the thermal resistance concept is illustrated in Fig. 4. The following assumptions are considered in the analytical model.

1. A one-dimensional steady-state heat transfer model is considered. The solar irradiation and temperature parameters are regarded as constant and uniform.

2. Thermal losses due to convection and radiation are considered only at the top and bottom surfaces, as shown in Fig. 4, while the thermal losses through the side walls in PV and TEGs are neglected.

3. The contact resistance between the adjacent layers of the hybrid PV/TEG system is neglected.

4. The thermal loss due to radiation between the heat sink and ground is negligible.

5. The properties of the $\mathrm{p}-\mathrm{n}$ leg semiconductors in TEG are considered as constant and independent of temperature.

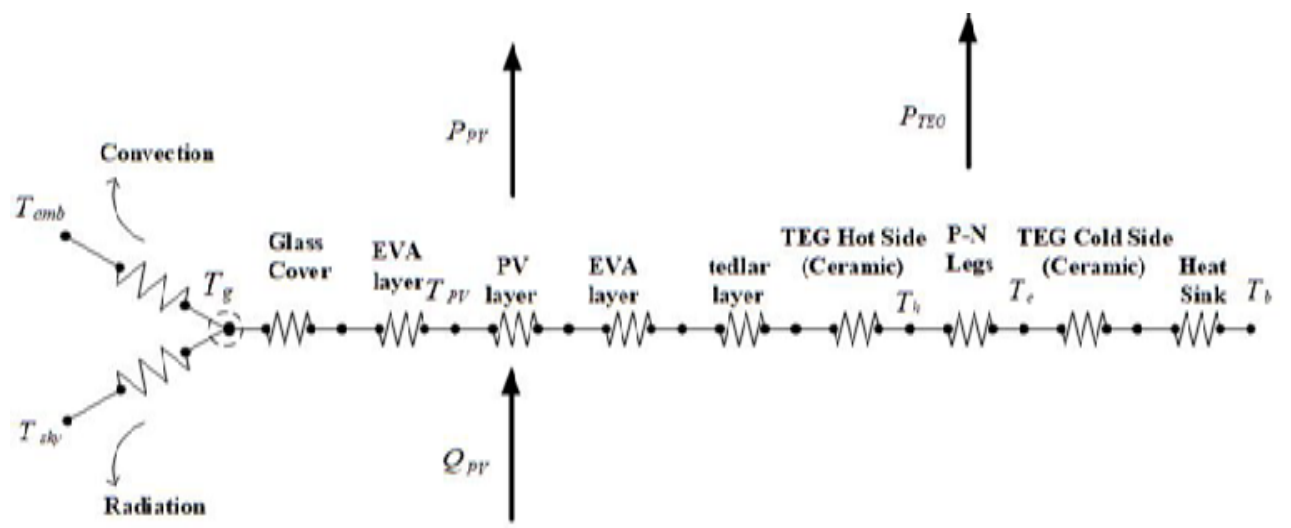

Fig. 4. Equivalent thermal circuit of the PV/TEG system under study 


\subsection{Photovoltaic panel}

The solar energy received by the PV layer $\left(Q_{P V}\right)$ is given as

$$
Q_{P V}=\tau_{g}\left[\alpha_{P V} \beta_{P V}+\alpha_{T}\left(1-\beta_{P V}\right)\right] G A_{P V}
$$

where $\tau_{g}$ is the transmissivity of the glass layer, $\alpha_{P V}$ is the absorptivity of the PV, $\beta_{P V}$ is the packaging factor of the PV module, $\alpha_{T}$ is the absorptivity of the tedlar, $G$ is the solar radiation intensity and $A_{P V}$ is the area of the PV module.

Thermal loss due to conduction and convection $\left(Q_{\text {con } / \text { conv }}\right)$ from the PV layer to the ambient air through the glass layer is given as

$$
\begin{aligned}
& Q_{\text {con } / \text { conv }}=U_{t}\left(T_{P V}-T_{a m b}\right) A_{P V} \\
& U_{t}=\left[\frac{L_{g}}{K_{g}}+\frac{1}{h_{\text {air }}}+\frac{L_{E V A}}{K_{E V A}}\right]^{-1} \\
& h_{\text {air }}=2.8+3.0 \mathrm{~V}
\end{aligned}
$$

where $U_{t}$ is the heat transfer coefficient due to conduction and convection from the PV layer to ambient air. $T_{P V}$ is the PV temperature, $T_{a m b}$ is the ambient temperature, $L_{g}$ and $L_{E V A}$ are the thickness of the glass and EVA layer and $K_{g}$ and $K_{E V A}$ are the thermal conductivity of glass and EVA layer, respectively. The natural convective heat transfer coefficient $\left(h_{\text {air }}\right)$ is calculated using eq. 4 , where $V$ is wind velocity.

The thermal loss due to radiation from the PV to the surroundings is estimated as

$$
\begin{aligned}
& Q_{\text {rad }}=\varepsilon_{g} \sigma A_{P V}\left[T_{P V}^{4}-T_{s k y}^{4}\right] \\
& T_{\text {sky }}=0.0552 T_{a m b}^{1.5}
\end{aligned}
$$

where $\varepsilon_{g}$ is the emissivity of glass, and $\sigma$ is the Stefan - Boltzmann constant, $5.67 \times 10^{-8}$ $\mathrm{W} / \mathrm{m}^{2} \mathrm{~K}^{4}$ and $T_{\text {sky }}$ is the sky temperature.

The power generated by $\mathrm{PV}\left(P_{P V}\right)$ is given as

$$
P_{P V}=\tau_{g} \beta_{P V} \eta_{P V} G A_{P V}
$$

where $\eta_{P V}$ is the efficiency of the PV module, which can be determined as given in eq. (8).

$$
\eta_{P V}=\eta_{\text {ref }}\left[1-\gamma\left(T_{P V}-T_{P V, r e f}\right)\right]
$$


here $\eta_{\text {ref }}$ is the reference PV efficiency under standard test conditions (STC): solar irradiation $G_{\text {ref }}=1000 \mathrm{~W} / \mathrm{m}^{2}$, reference PV temperature $T_{P V \text {,ref }}=25^{\circ} \mathrm{C}$ and air mass $A M=1.5$ and $\gamma$ is the temperature coefficient.

The amount of heat transferred to the hot side of the TEG is given as

$$
\begin{aligned}
Q_{h} & =U_{T}\left(T_{P V}-T_{h}\right) \\
U_{T} & =\left[\frac{L_{P V}}{K_{P V} A_{P V}}+\frac{L_{E V A}}{K_{E V A} A_{P V}}+\frac{L_{\text {tedlar }}}{K_{\text {tedlar }} A_{P V}}+\frac{L_{\text {ceramic }}}{K_{\text {ceramic }} A_{T E G} N_{T E G}}\right]^{-1}
\end{aligned}
$$

The overall heat transfer from the PV layer to the TEG hot side is expressed based on energy balance as

$$
Q_{P V}=P_{P V}+Q_{\text {con/conv }}+Q_{\text {rad }}+Q_{h}
$$

\subsection{Thermoelectric generator}

The energy received on TEG hot side $\left(Q_{h}\right)$ and TEG cold side $\left(Q_{c}\right)$ is also given as shown in eq. 11 and 12.

$$
\begin{aligned}
& Q_{h}=\alpha_{p n} I T_{h}+\left[T_{h}-T_{c}\right] K-0.5 I^{2} R \\
& Q_{c}=\alpha_{p n} I T_{c}+\left[T_{h}-T_{c}\right] K+0.5 I^{2} R
\end{aligned}
$$

where $\alpha_{p n}$ is the Seebeck coefficient, $R$ and $K$ is internal resistance and heat transfer coefficient respectively, and can be calculated as given in eq. $\alpha_{p n} I T_{h}$ and $\alpha_{p n} I T_{c}$ represents the Peltier heat on the hot and cold junction of the TEG. $\left[T_{h}-T_{c}\right] K$ is the Newton heat transfer and $0.5 I^{2} R$ is the Joule heat.

$$
\begin{aligned}
& R=\frac{N_{p n} \rho_{p} L_{p}}{A_{p}}+\frac{N_{p n} \rho_{n} L_{n}}{A_{n}} \\
& K=\frac{N_{p n} K_{p} A_{p}}{L_{p}}+\frac{N_{p n} K_{n} A_{n}}{L_{n}}
\end{aligned}
$$

Parameters with ' $\mathrm{p}$ ' subscript refer to P-type semiconductor leg, and parameters with ' $\mathrm{n}$ ' subscript refer to N-type semiconductor leg. $N_{p n}$ is the number of pairs of P and N-type semiconductor legs in the TEG, $\rho, L, K$ and $A$ is the electrical resistance, length, section area, and heat coefficient of P-N legs. Now the output power of TEG is given as

$$
P_{T E G}=Q_{h}-Q_{c}=I \alpha_{p n}\left(T_{h}-T_{c}\right)-I^{2} R=I^{2} R_{L}
$$

where $R_{L}$ is the load resistance. TEG output is maximum when the load resistance is equal to the internal resistance of TEG. Hence, the current $I$ flowing through the TEG is given as 


$$
I=\frac{\alpha_{p n}\left[T_{h}-T_{c}\right]}{2 R}
$$

The amount of energy leaving the TEG cold side is also given as,

$$
Q_{c}=\frac{T_{c}-T_{b}}{R_{h s}}
$$

where $T_{b}$ is the heat sink rear boundary temperature which is considered as ambient temperature for natural convection.

\subsection{Heat Sink}

A pin-fin type heat sink is used to cool the cold junction of the TEG. The resistance of heat sink $R_{h s}$ is the sum of thermal resistance of the heat sink base $R_{b}$ and the thermal resistance of heat sink fins $R_{f i n} . N_{T E G}$ denotes the number of TEG or number of heat sinks used.

$$
\begin{aligned}
& R_{h s}=R_{b}+R_{\text {fin }} \\
& R_{b}=\frac{L_{b}}{K_{b} A_{b} N_{T E G}} \\
& R_{\text {fin }}=\frac{1}{\eta_{o} h_{\text {air }} A_{\text {full }}}
\end{aligned}
$$

where $L_{b}, K_{b}$ and $A_{b}$ is the thickness, thermal conductivity and area of the heat sink base. $\eta_{o, f i n}$ is the efficiency of all the fins, $A_{f u l l}$ is the fin full surface area and they are given as

$$
\begin{aligned}
& A_{\text {full }}=N_{T E G}\left(n_{\text {fin }} A_{\text {fin }}+A_{b}\right) \\
& \eta_{\text {fin }}=\frac{\tanh \left(m H_{c_{\text {f } \text { fin }}}\right)}{m H_{c, \text { fin }}} \\
& m=\sqrt{\frac{4 h_{\text {air }}}{K_{\text {fin }} D_{\text {fin }}}} \\
& H_{c_{\text {f } \text { fin }}}=H_{\text {fin }}+D_{\text {fin }} / 4 \\
& \eta_{\text {o,fin }}=1-\frac{n_{\text {fin }} N_{\text {TEG }} A_{\text {fin }}}{A_{\text {full }}}\left(1-\eta_{\text {fin }}\right) \\
& A_{\text {fin }}=\pi D_{\text {fin }} H_{c_{\text {ffin }}}
\end{aligned}
$$


where $n_{f i n}$ is the efficiency of single fin, $A_{\text {fin }}$ is the cross-sectional area of the fin, $D_{f i n}$ is the diameter of the fin, $H_{\text {fin }}$ is the height of the fin and $H_{c, f i n}$ is the corrected fin length.

\section{Result and Discussion}

\subsection{Performance analysis}

The performance evaluation of the hybrid PV/TEG system is calculated for a day in the summer season from morning 7.00 am to the evening at $7.00 \mathrm{pm}$ (March 15, 2020). The average solar radiation received on the dual-axis tracking PV panel calculated using TracePro is considered (see Fig. 5). The ambient temperature and wind velocity are considered constant and assumed as $300 \mathrm{~K}$ and $1.8 \mathrm{~m} / \mathrm{s}$, respectively, since the present study focuses only on the effect of tracking and non-tracking condition on the hybrid PV/TEG system's performance. For the first simulation, the number of TEGs on the PV panel's rear side is varied from 50 to 450 TEGs.

Fig 6. Shows the variation of $P_{T E G}$ versus one day solar irradiance for 50 to 450 TEGs. It is evident from the analysis that the $P_{T E G}$ increases when there is an increase in solar radiation. Also, the $P_{T E G}$ increases when the number of TEG modules is increased from 50 to 350 and again $P_{T E G}$ decreases when the number of TEG modules is greater than 350 . This is mainly because when the number of TEG has increased, the contact area between PV and TEG increases, which reduces the temperature difference between TEG hot side and TEG cold side leading to a drop-in $P_{T E G}$. Fig. 7 clearly shows that when the number of TEG on the rear side of the PV panel is increased, it reduces the temperature difference between TEG hot and cold side, thus affecting the power output of the TEG.

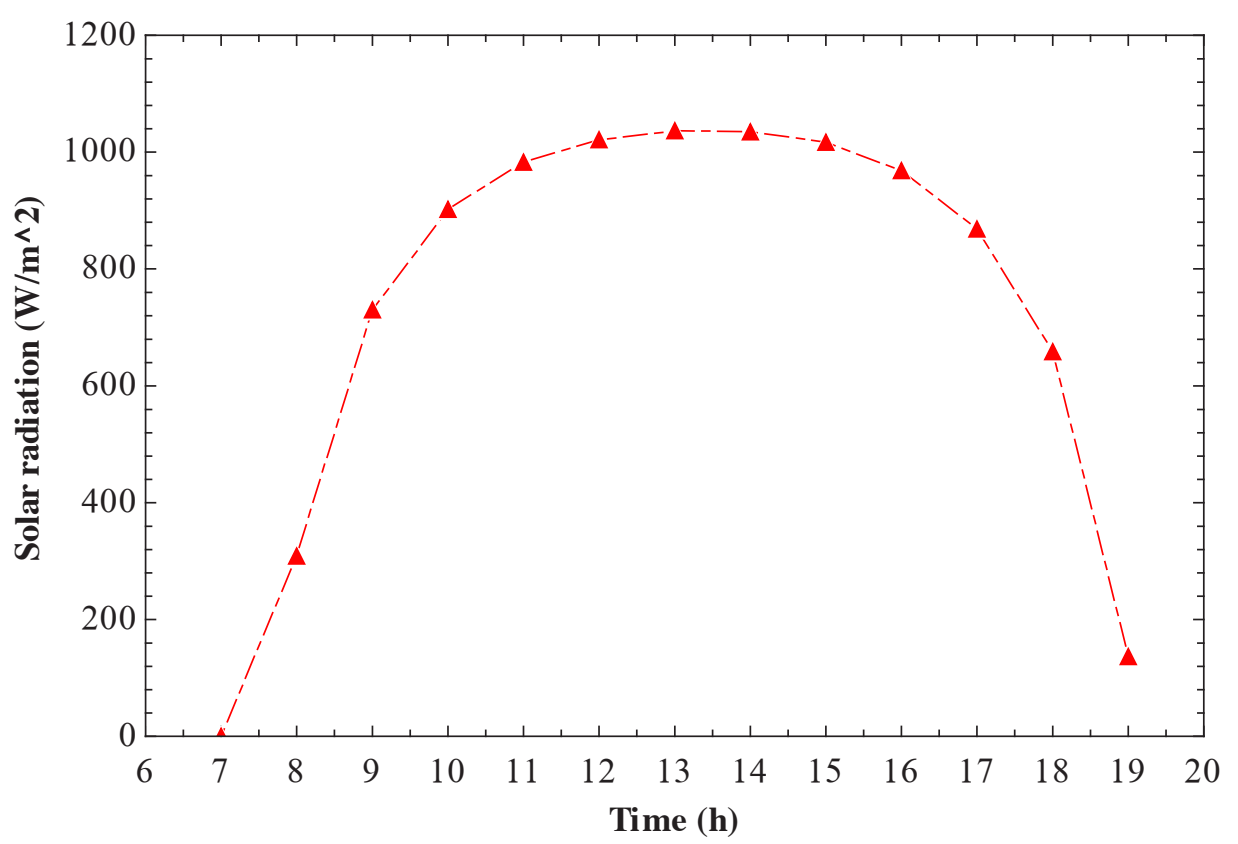

Fig. 5. Solar radiation received on March 15, 2020 (dual-axis tracking) 


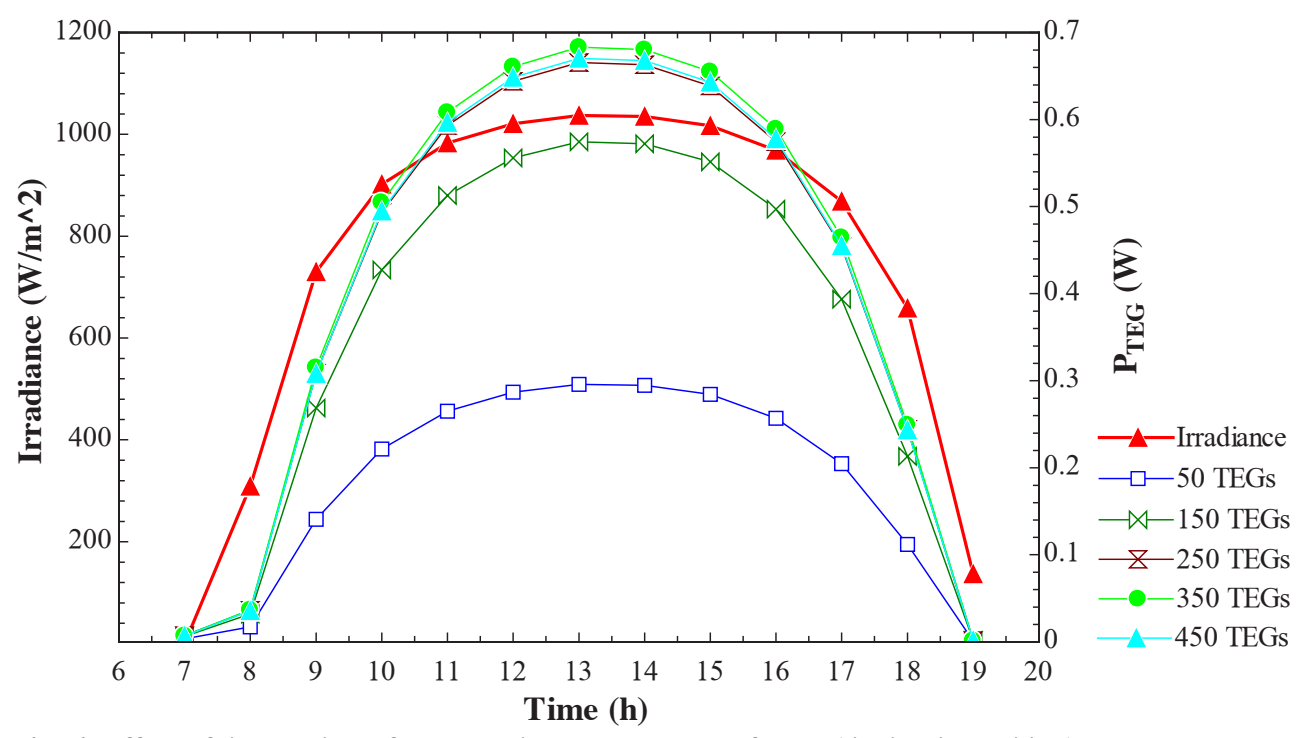

Fig. 6. Effect of the number of TEG on the power output of TEG (dual-axis tracking)

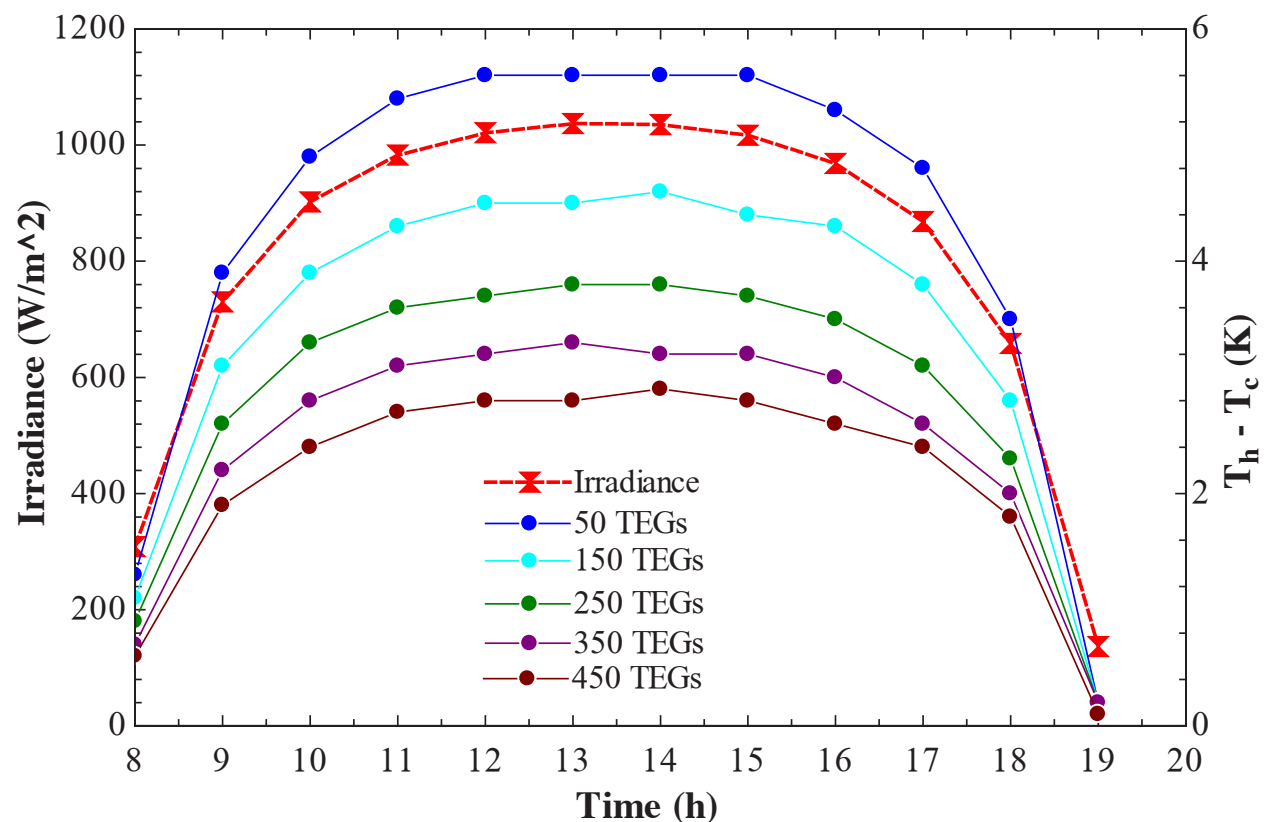

Fig. 7. Effect of number of TEG modules on the temperature difference between TEG hot and cold junction (dual-axis tracking)

Fig. 8 shows the variation of PV temperature for varying solar radiation and the number of TEGs. As expected, the value of $T_{P V}$ increase when the solar radiation is higher, and it reduces as the number of TEGs is increased on the rear side of the PV. For a fixed heat sink and a constant wind velocity the $T_{P V}$ reduces by increasing the number of TEGs, which results in less efficiency of individual TEG. Although individual TEG would be less efficient, the total power output of all TEGs together will increase up to a certain number 
(in Fig. 6, the total $P_{T E G}$ increases till 350 TEGs above 350 TEGs the $P_{T E G}$ again decreases). Hence optimisation of the number of TEGs is required in a hybrid PV/TEG $\left(P_{P V / T E G}\right)$ system for maximum power output from TEG. The effect of the number of TEGs on the overall power output of PV/TEG is given in Fig. 9. It is found that the increase in the number of TEGs increases the overall power output of PV/TEG. Although the rise in the number of TEG above a certain limit affects the $P_{T E G}$ on the other hand, it reduces the PV cell temperature, which improves the efficiency of PV. Hence, the increase in TEG modules lead to an increase in $P_{P V}$ which lead to an increase in $P_{P V / T E G}$.

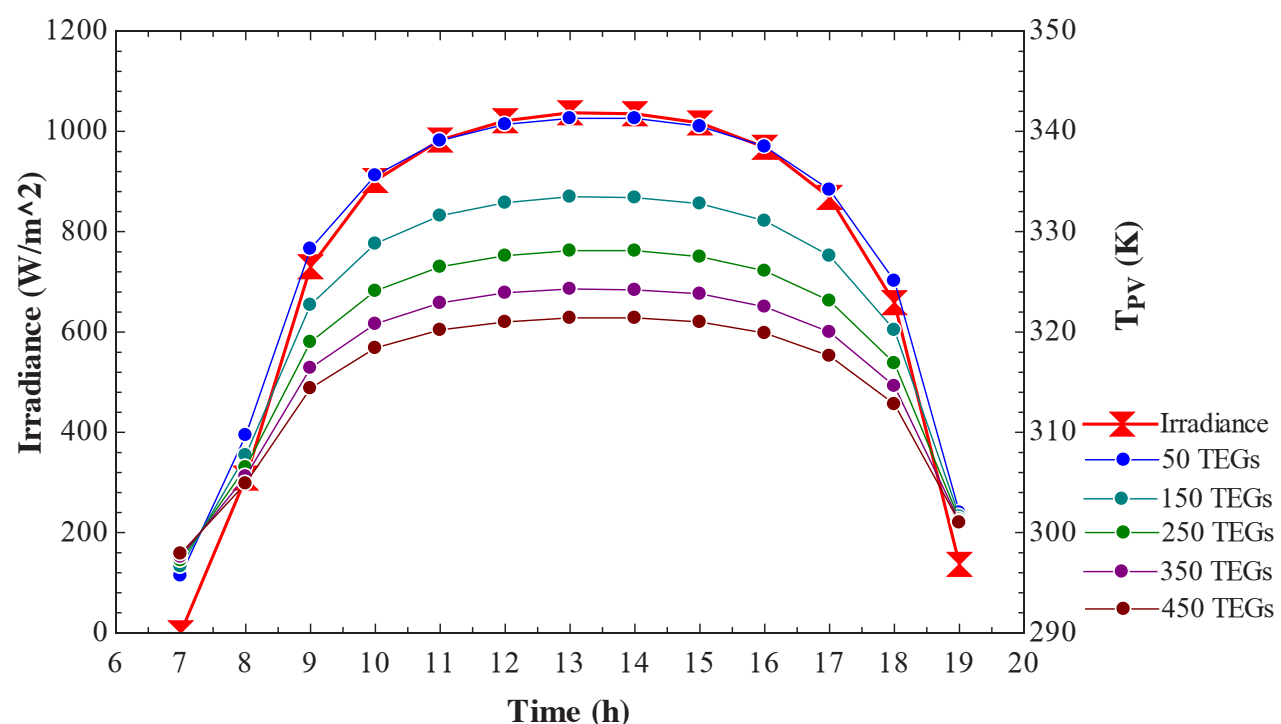

Fig. 8. Effect of number of TEG modules on the PV module temperature (dual-axis tracking)

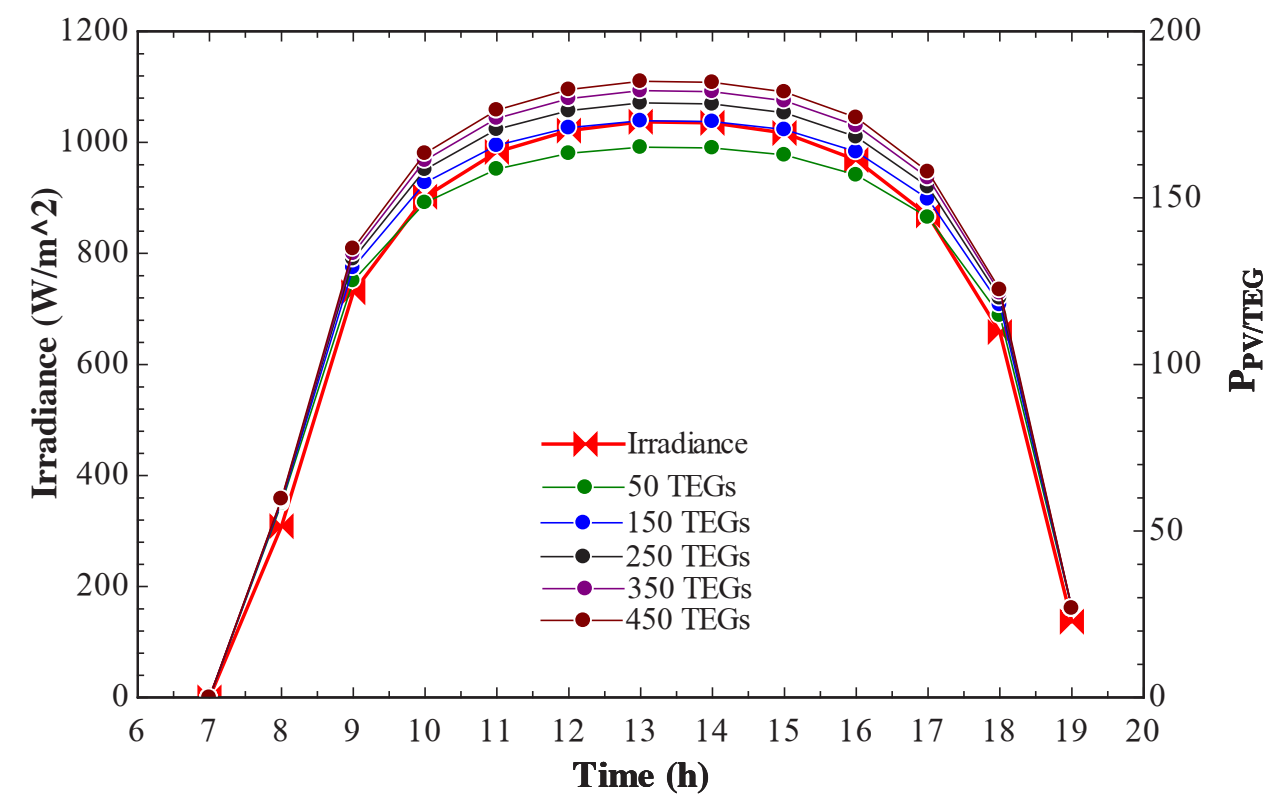

Fig. 9. Effect of number of TEG on the PV power output (dual-axis tracking) 


\subsection{Optimisation of TEG modules under tracking and no-tracking condition}

In this work, the performance and effect of the number of TEGs on a hybrid PV/TEG system's performance are studied for a summer day under dual-axis tracking conditions. However, it's important to study the effect of tracking and non-tracking on the PV/TEG system's performance and determine the optimum number of TEG modules for maximum output from TEGs. For the TracePro simulation, hourly solar radiation (from 8.00 am to $7.00 \mathrm{pm})$ is considered for one day $\left(15^{\text {th }}\right.$ of every month) in every month of the year 2020 . From this, the average monthly solar irradiance received on the PV panel is calculated using TracePro, as shown in Fig. 10. Fig. 10 shows that the dual-axis tracking PV panel receives more stable irradiance compared with no-tracking and single-axis tracking.

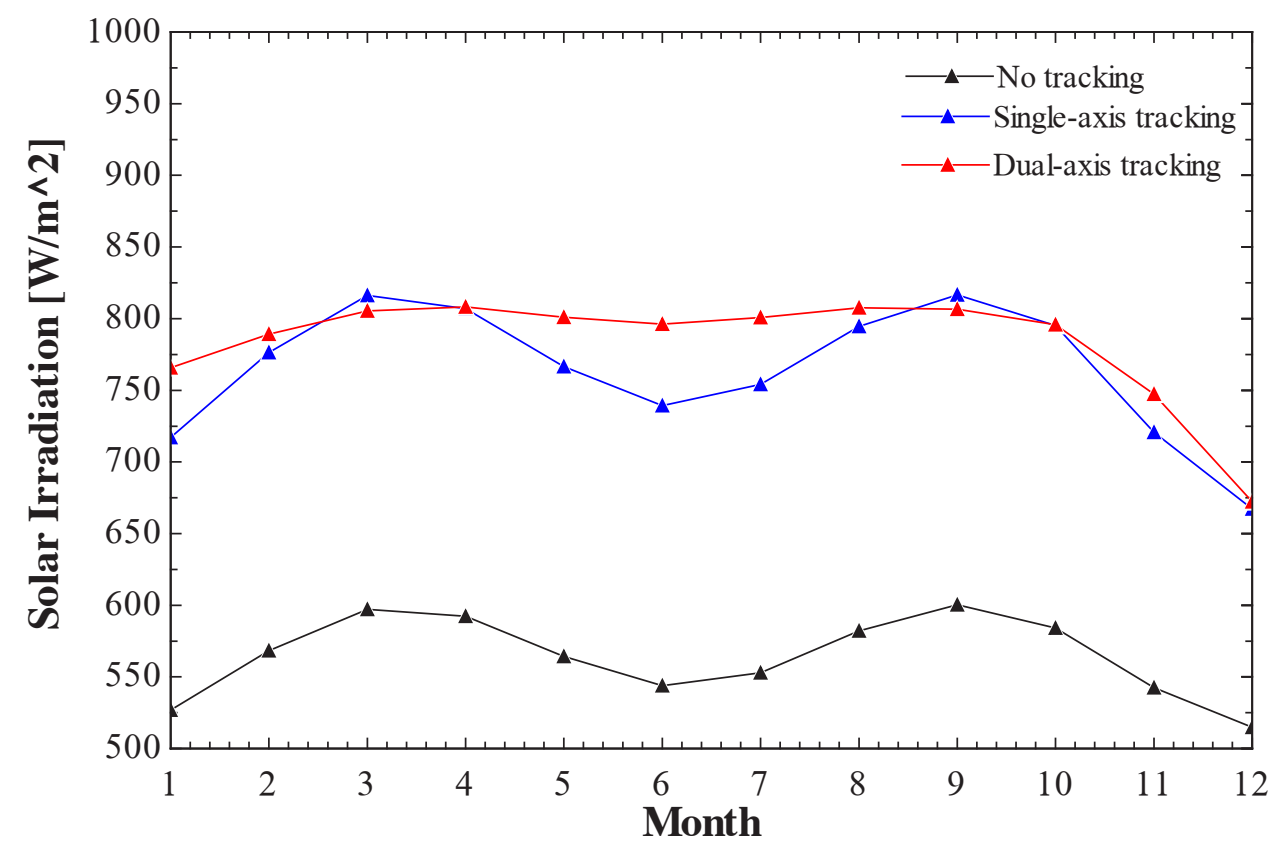

Fig. 10. The average solar irradiance distribution under tracking and non-tracking condition

For optimising the number of TEG modules, solar radiation at 12 instances with 1-hour interval is considered for a day in every month. Totally 144 instances were considered for the year 2020, and the power output of TEG is calculated at these instances to optimise the number of TEG modules. Using quadratic approximation method in EES, the obtained optimum number of TEGs for maximum net $P_{T E G}$ are 336, 339, and 341 for no-tracking, single-axis, and dual-axis tracking conditions, respectively. Considering the obtained optimum number of TEGs, the TEG and PV's power output is determined at 144 instances (See Fig. 11 and 12). Fig. 11 shows the distribution of the 144 instances among the various TEG power output ranges for the three tracking scenarios. It is evident from the graph that the case of dual-axis tracking has the highest instance count for the maximum power range, followed by the single-axis tracking and no tracking cases. Considering the lower power range, the instance count trend is reversed, with the no tracking case having the greatest number of lowest TEG outputs. Considered holistically, this distribution shows that since the total count of instances for each case is the same, the dual-axis tracking and single-axis tracking cases consist of a larger proportion of high TEG instances compared to the no tracking case, which in turn is majorly made up of low TEG output instances. This 
observation aligns well with the tracking performance's initial inference - dual-axis tracking maximises the irradiation falling on the setup followed by single-axis tracking compared to the no tracking case. Higher irradiation, in turn, translates to higher net $P_{T E G}$ values, which is what is illustrated in Fig. 11.

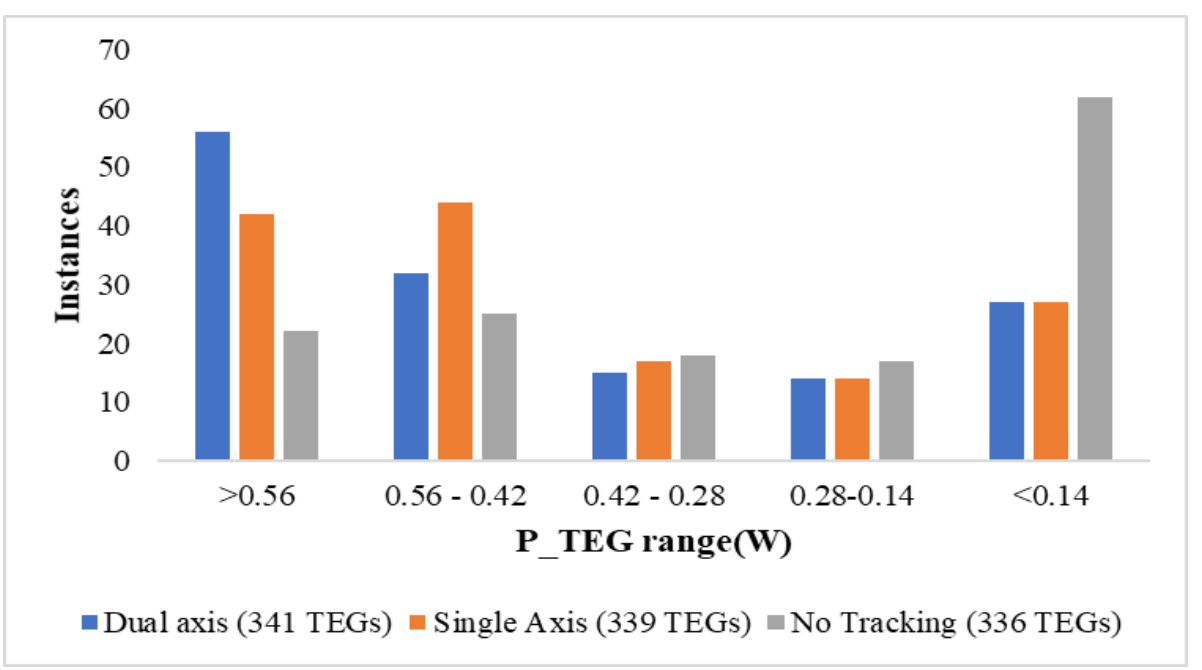

Fig. 11. Histogram of power produced by the optimum number of TEGs in the PV/TEG system

Fig. 12 illustrates the distribution of the 144 instances of PV power for each tracking scenario. The overall trend observed is similar to the case of the distribution count of the $P_{T E G}$ with the dual-axis tracking case having the maximum net $P_{P V}$ since it consists of a larger proportion of high PV power instances. The reason for this trend is also quite similar - the discrepancy in the tracking performance in the three cases leads to higher net irradiation in the dual-axis case followed by the single axis and the no tracking cases, respectively, which in turn results in proportionately higher net PV output.

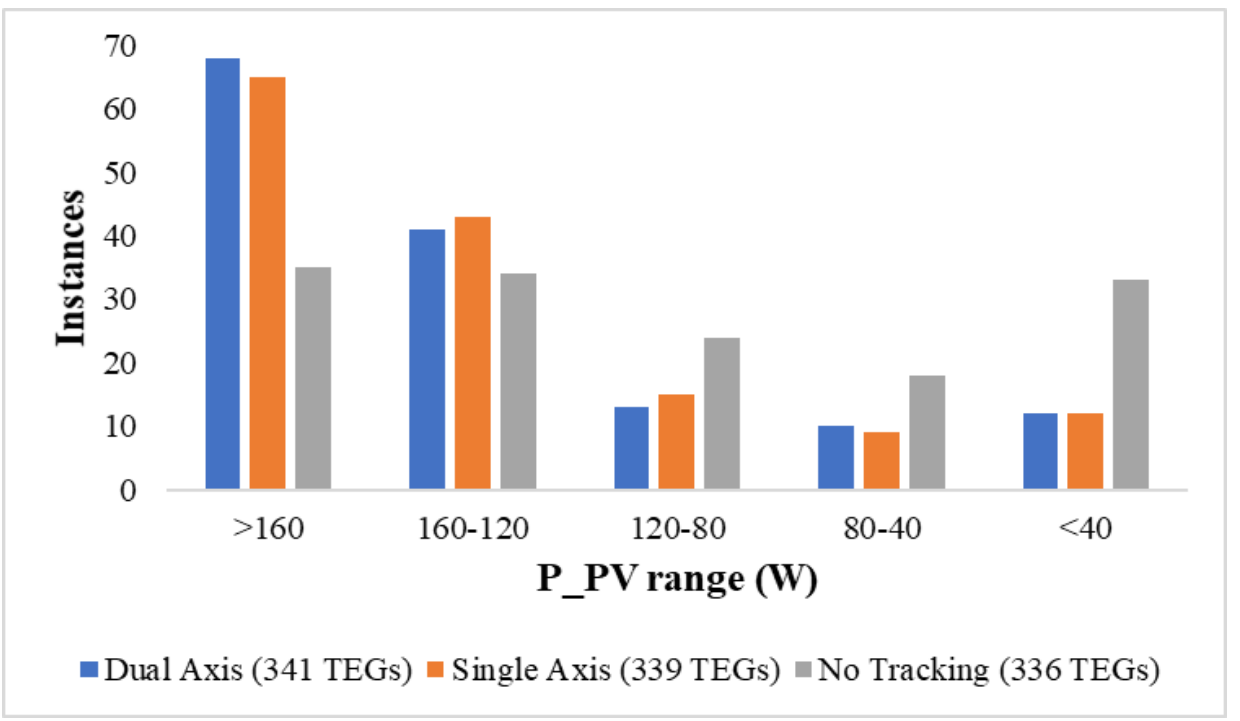

Fig. 12. Histogram of power produced by the PV when using the optimum number of TEGs in PV/TEG system 


\section{Conclusion}

In the present study, a hybrid PV/TEG system's performance under tracking and the notracking condition is simulated via EES. The TEG modules are integrated at the PV panel's rear side to make use of the excess PV temperature. It is shown that higher solar radiation leads to higher temperature differences in TEG, which would lead to higher TEG output. However, increasing the number of TEG modules will decrease the temperature difference across the TEG, leading to lower TEG output. Although increasing the TEG modules reduces its power output, on the other hand, it helps to reduce the PV cell temperature and improves the PV output. So, if we optimise the number of TEG modules for maximum PV output in a hybrid PV/TEG, it would require more TEG modules, thus increasing the overall cost of the system. In the present study, the number of TEGs in a hybrid PV/TEG system is optimised under tracking and no-tracking condition for maximum net TEG output performance

The obtained optimum number of TEGs for the maximum net $P_{T E G}$ are 336, 339, and 341 for no-tracking, single-axis, and dual-axis tracking conditions, respectively. It is shown that the dual-axis tracking PV/TEG with 341 number of TEGs generates maximum net TEG output and maximum net PV output at most of the instances compared with singleaxis tracking (339 TEGs) and no-tracking (336 TEGs). Therefore, if TEG's output power is considered in a PV/TEG system under constant ambient temperature and wind velocity, the optimum number of TEG can be determined, as mentioned in this study. However, for a large scale PV/TEG design, among several factors, the cost of the system should also be considered in determining the optimum number of TEGs on the rear side of the PV module.

Acknowledgement: Taylor's University supported this work through its TAYLOR'S Ph.D. SCHOLARSHIP Programme through grant TUFR/2017/001/01.

\section{References}

1. N. Jaziri, A. Boughamoura, J. Müller, B. Mezghani, F. Tounsi, Energy Rep. (2019)

2. D. Beretta, N. Neophytou, J.M. Hodges, M.G. Kanatzidis, D. Narducci, M. MartinGonzalez, M. Beekman, B. Balke, G. Cerretti, W. Tremel, A. Zevalkink, A.I. Hofmann, C. Müller, B. Dörling, M. Campoy-Quiles, M. Caironi, Mater. Sci. Eng. Rec. Rep. 138, 210 (2019)

3. P. Huen, W. A. Daoud, Renew. Sustain. Energy Rev. 72, 1295 (2017)

4. G. Li, S. Shittu, T.M.O. Diallo, M. Yu, X. Zhao, J. Ji, Energy. 158, 41 (2018)

5. A. Z. Sahin, K. G. Ismaila, B. S. Yilbas, A. Al-Sharafi, Int. J. Energy Res. 44, 3365 (2020)

6. A. Allouhi, Sol. Energy Mater. Sol. Cells. 200, 109954 (2019)

7. P. D. Raut, V.V. Shukla, S. S. Joshi, Int. J. Eng. Technol. 7, 2619 (2018)

8. S. Sripadmanabhan Indira, C. A. Vaithilingam, K. K. Chong, R. Saidur, M. Faizal, S. Abubakar, S. Paiman, Sol. Energy. 201, 122 (2020)

9. C. Babu, P. Ponnambalam, Energy Convers. Manag. 173, 450 (2018)

10. W. Gu, T. Ma, A. Song, M. Li, L. Shen, Energy Convers. Manag. 198, 111800 (2019)

11. C. Haiping, H. Jiguang, Z. Heng, L. Kai, L. Haowen, L. Shuangyin, Energy. 166, 83 (2019)

12. S. Shittu, G. Li, Y. G. Akhlaghi, X. Ma, X. Zhao, E. Ayodele, Renew. Sustain. Energy Rev. 109, 24 (2019) 
13. R. Bjørk, K.K. Nielsen, Sol. Energy. 120, 187 (2015)

14. E. A. Chávez-Urbiola, Y.V. Vorobiev, L.P. Bulat, Sol. Energy. 86, 369 (2012)

15. H. Najafi, K. A. Woodbury, J. Sol. Energy Eng. Trans. ASME. 135, 1 (2013)

16. D. Li, Y. Xuan, Q. Li, H. Hong, Energy. 126, 343 (2017)

17. O. F. Marandi, M. Ameri, B. Adelshahian, Sol. Energy. 161, 38 (2018)

18. L. Kütt, J. Millar, M. Lehtonen, M. Märss, RTUCON, 3 (2015) 